Check for updates

Cite this: RSC Adv., 2017, 7, 40392

\title{
Synthesis of chelating polyamine fibers and their adsorption properties for nickel(II) ions from aqueous solution
}

\begin{abstract}
Jianlan Cui, $\uparrow$ Yanru Li, $\mathbb{D} \dagger^{\star}$ Jian Meng, Congsan Zhong and Peng Wang
A novel fiber supported adsorbent PVAF-g-PEI was first developed by grafting polyethylenimine on polyvinyl alcohol fibers (PVAF). In order to increase the nickel(II) adsorption capacity of PVAF-g-PEI, they were chemically modified with amine-rich reagent tetraethylenepentamine by using a crosslinker, glutaraldehyde (GA). The effect of grafting reaction parameters was investigated. The prepared fiber sorbent PA-PVAF was characterized by using FT-IR, SEM, XPS and element analysis and used for the adsorption of nickel(॥) from aqueous solutions. The factors influencing nickel(॥) adsorption on PA-PVAF, such as contact time, solution $\mathrm{pH}$, temperature and ionic strength, were also studied. At $\mathrm{pH} 7.0$, the sorption capacity of PA-PVAF was $91.03 \mathrm{mg} \mathrm{g}^{-1}$ for nickel(I). The Langmuir isotherm model precisely explained the equilibrium data. Adsorbent-adsorbate kinetics were pseudo second order. Meanwhile, the adsorption-desorption cycle experiments showed that PA-PVAF had good reuse performance.
\end{abstract}

Received 27th June 2017 Accepted 25th July 2017

DOI: 10.1039/c7ra06935a

rsc.li/rsc-advances

exchange, membrane filtration, chemical reduction and reverse osmosis. ${ }^{6-9}$ Due to the advantages of low running cost, low dissipation of energy and easy operation, the adsorption method has received much attention. ${ }^{10-12}$

Krishnan et al. ${ }^{10}$ reported a kind of activated carbon adsorbent prepared from raw sugarcane bagasse pith. The adsorption experiments showed high adsorption capacity of $140.85 \mathrm{mg} \mathrm{g}^{-1}$ for the removal of $\mathrm{Ni}$ (II) from aqueous solutions at $\mathrm{pH}$ 6.5. Meanwhile, the activated carbon adsorbent showed high adsorption capacity, reusability, low cost and environmental protection. Euvrard et al. ${ }^{11}$ prepared two new silica-based sorbents. At $\mathrm{pH} 7.0$, the maximum adsorption capacity for $\mathrm{Ni}(\mathrm{II})$ on the adsorbent was found to be $182 \mathrm{mg} \mathrm{g}^{-1}$ and $210 \mathrm{mg}$ $\mathrm{g}^{-1}$ respectively. Apart from high sorption capacities, these sorbents presented additional advantages such as cost effective

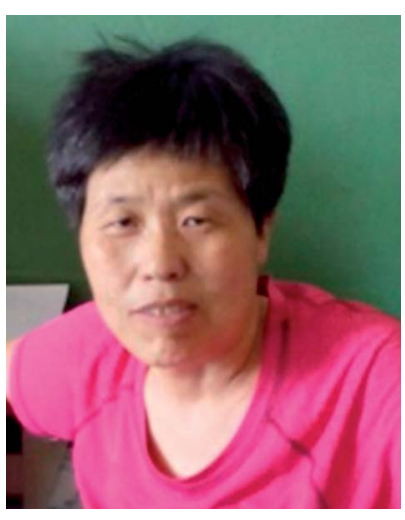

Jianlan Cui: Female, fifty years old, Shanxi Linfen person, Professor, School of chemical engineering and the environment, North University of China, director of the Department of Chemical Engineering, Director of instrument testing center. Mainly engaged in the pharmaceutical, functional polymer materials and fine chemical industry research. Communication e-mail: 414559203@qq.com

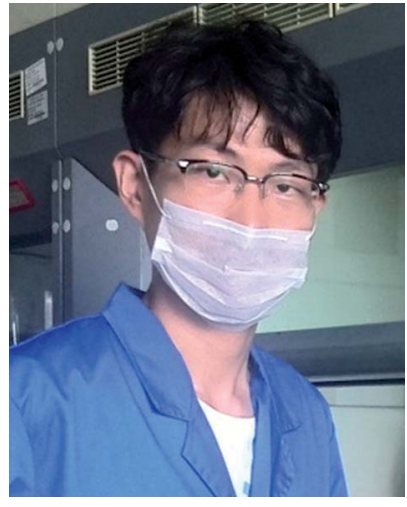

Jian Meng: Male, twenty-seven years old, Shanxi Huairen person, Doctoral candidate, School of chemical engineering and the environment, North University of China, Shanxi, China. Mainly engaged in the functional polymer materials and fine chemical industry research. Communication $e$ mail:844986037@qq.com 


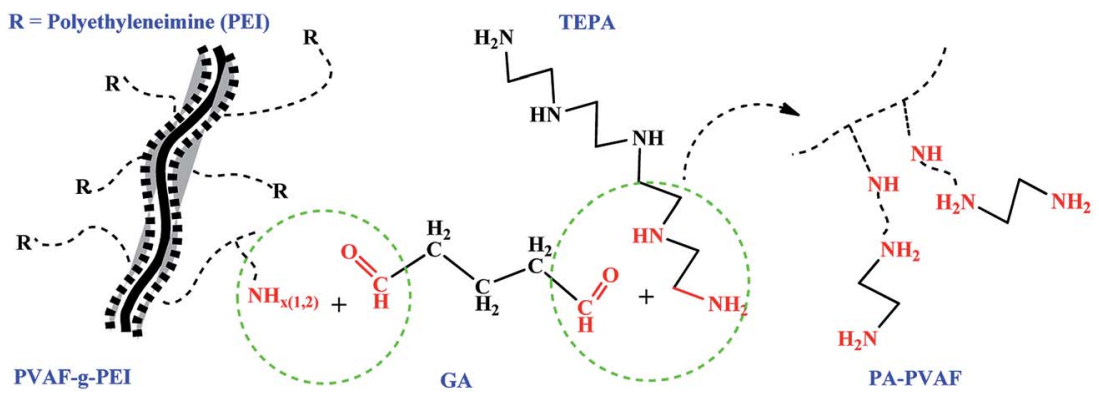

Scheme 1 Chemistry of preparing functional fibers PA-PVAF.

and facile preparation. Wakeel et al. ${ }^{12}$ performed an investigation on removal of $\mathrm{Ni}$ (II) by nano magnetite chitosan films. The prepared films were tested for removal of $\mathrm{Ni}(\mathrm{II})$ ions in aqueous solution. The maximum removal percentage for sorption $\mathrm{Ni}(\mathrm{II})$ was $91 \%$ using the composite films at $\mathrm{pH}$ 6.5. Moreover, the sorption-desorption studies showed good recyclability of the films.

Polyethylenimine (PEI) is a kind of heavy metal chelating agent. There are a large number of amino groups on the molecular chain, which makes it have strong complexing ability for heavy metal ions. It has wide application prospect in the treatment of wastewater containing heavy metal and dye. ${ }^{13,14}$ However, because PEI is soluble in water, it is difficult to recover it if directly used for adsorption, whereas immobilizing the PEI on solid support can resolve that. ${ }^{15}$ At the same time, no secondary pollution should be taking into account in the removal of pollutants.

In this study, with an effort to prepare an adsorbent with high efficiency, no secondary pollution and easy operation, polyvinyl alcohol fibers (PVAF) were selected as carrier. First, the PEI chain was grafted onto PVAF surface with the aid of coupling agent $\gamma$-glycidoxypropyltrimethoxysilane, obtaining grafted fiber PVAF- $g$-PEI. Then, the polyamine compound tetraethylenepentamine was bonded to the surface of the PVAF- $g$ PEI fibers using crosslinking agent glutaraldehyde, and the polyamine chelating fiber (PA-PVAF) was prepared. The specific process was presented in Scheme 1. Furthermore, the adsorption behavior of PA-PVAF to Ni(II) was investigated. The results indicated that the PA-PVAF was an efficient fibrous sorbent for the effective removal of Ni(II). The polyamine adsorbent PAPVAF not only has good adsorption performance, but also has the advantages of environmental protection. It will not cause secondary pollution to the environment after be abandoned.

\section{Experimental section}

\subsection{Reagents and instruments}

Polyvinyl alcohol fiber (PVAF, SY-9, Sichuan Vigny fiber factory); $\gamma$-glycidoxypropyltrimethoxysilane (GPTMS), polyethyleneimine (PEI, $50 \mathrm{wt} \%$ aqueous solution), glutaraldehyde (GA, 50\% in water), tetraethylenepentamine (TEPA), nickel nitrate were purchased from Shanghai Aladdin Chemistry Co. Ltd. (Shanghai, China); other reagents were pure chemical reagents. Deionized water was produced by our own. The glassware used in the experiments was soaked in $0.1 \mathrm{M} \mathrm{HNO}_{3}$ solution for $12 \mathrm{~h}$, then washed several times with deionized water, dried for standby.

Thermo Nikolet 6700 Infrared Spectrometer (FT-IR, Nicolet Company, America); S4800 Scanning Electron Microscope (SEM, Hitachi company, Japan); Axis UTLTRADLD X-ray photoelectron spectrometer (XPS, Shimadzu company, Japan); Elemental Analyzer (EA, Elementar Vario EL, Germany); AA6300C Atomic Absorption Spectrometer (AAS, Hitachi company, Japan).

\subsection{Preparation and characterization of functional chelating fiber PA-PVAF}

A certain amount of PVA fiber was placed in acetone solvent ultrasonic cleaning for half an hour, drying for standby.

(1) $1.0 \mathrm{~g}$ treated fiber was placed into $50 \mathrm{~mL}$ isopropanol, while adding $8 \mathrm{~mL}$ GPTMS, the reaction was performed at $65^{\circ} \mathrm{C}$ for $10 \mathrm{~h}$. The reaction products GPTMS/PVAF were washed several times with ethanol to remove unreacted silane physically adsorbed on the fiber surface, and then vacuum dried to constant weight.

(2) The product obtained in step one was placed in a flask containing $50 \mathrm{~mL}$ isopropanol. Then $5 \mathrm{~mL} 50 \mathrm{wt} \%$ PEI solution was added into the flask and adjusted the solution $\mathrm{pH}$ to 10 by using $\mathrm{Na}_{2} \mathrm{CO}_{3}$ solution. The grafting reaction was carried out at $50{ }^{\circ} \mathrm{C}$ for $12 \mathrm{~h}$. The products PVAF- $g$-PEI were repeatedly washed with distilled water to neutral, and vacuum dried to constant weight.

(3) The resultant fibers in step two were placed in $50 \mathrm{~mL}$ of water was added. Followed by the addition of $3 \mathrm{~mL}$ TEPA and stirring for $30 \mathrm{~min}$. Afterwards, $8 \mathrm{~mL} 3 \mathrm{wt} \%$ GA solution was added using constant pressure dropping funnel with stirring in $30 \mathrm{~min}$. The crosslinking reaction was conducted at $40{ }^{\circ} \mathrm{C}$ for $6 \mathrm{~h}$. After the reaction was finished, the products PA-PVAF were separated by filtration and rinsed several times with distilled water, and dried under vacuum.

The chemical structure of the resultant products was characterized and confirmed by using FT-IR, EA and XPS. The changes of fiber morphology were observed by using SEM.

\subsection{Study on adsorption properties of chelate fiber PA-PVAF}

$500 \mathrm{mg} \mathrm{L}^{-1}$ of $\mathrm{Ni}(\mathrm{II})$ based solution was prepared by using $\mathrm{Ni}\left(\mathrm{NO}_{3}\right)_{2} \cdot 6 \mathrm{H}_{2} \mathrm{O}$, and different concentrations of $\mathrm{Ni}(\mathrm{II})$ were diluted from the basic solution. The concentration of $\mathrm{Ni}$ (II) in the solution was determined by using AAS and all the 


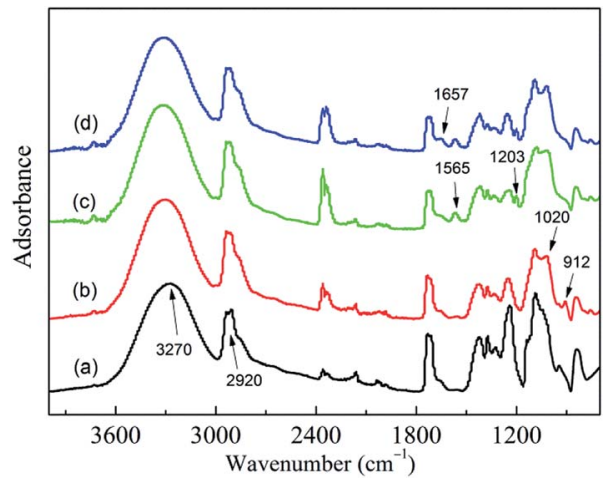

Fig. 1 IR spectra of fibers (a) PVAF, (b) GPTMS/PVAF, (c) PVAF-g-PEI, (d) PA-PVAF.

experiments were repeated three times. The $\mathrm{pH}$ value of $\mathrm{Ni}$ (II) solution was adjusted by $0.1 \mathrm{M} \mathrm{HCl}$ and $0.1 \mathrm{M} \mathrm{NaOH}$.

$300 \mathrm{mg} \mathrm{L}^{-1}$ of Ni(II) solution was prepared using the above based solution. PVAF- $g$-PEI and PA-PVAF were used as adsorbent, and the adsorption kinetics were carried out. The results showed that the adsorption equilibrium time was about $90 \mathrm{~min}$. $0.05 \mathrm{~g}$ PA-PVAF was introduced into $30 \mathrm{~mL} \mathrm{Ni(II)} \mathrm{solution} \mathrm{with}$ different concentration (from 30 to $300 \mathrm{mg} \mathrm{L}^{-1}$ ). The isothermal adsorption experiments were performed for $90 \mathrm{~min}$ in a constant temperature oscillator. Then the adsorbent was separated by filtration and the $\mathrm{Ni}$ (II) concentration in the solution was determined by using AAS. The equilibrium adsorption amount was calculated according to eqn (1.1). The desorption rate of $\mathrm{Ni}(\mathrm{II})$ in desorption experiment was calculated by using eqn (1.2).

$$
\begin{gathered}
Q_{\mathrm{e}}=\frac{V\left(C_{0}-C_{\mathrm{e}}\right)}{m} \\
\text { Desorption rate }=\frac{C_{\mathrm{d}} V_{\mathrm{d}}}{\left(C_{0}-C_{\mathrm{e}}\right) V}
\end{gathered}
$$

where $Q_{\mathrm{e}}\left(\mathrm{mg} \mathrm{g}^{-1}\right)$ is the equilibrium adsorption amount; $V$ and $V_{\mathrm{d}}(\mathrm{mL})$ the volume of the adsorption liquid and eluent, respectively; $C_{0}$ and $C_{\mathrm{e}}\left(\mathrm{mg} \mathrm{L}^{-1}\right)$ the initial and equilibrium concentration of $\mathrm{Cd}$ (II) solution, respectively, $C_{\mathrm{d}}\left(\mathrm{mg} \mathrm{L}^{-1}\right)$ the concentration of $\mathrm{Cd}(\mathrm{II})$ in the eluent, respectively.

\section{Results and discussion}

\subsection{Characterization of functional fibers}

Fig. 1 shows the FT-IR spectra of different fibers.

According to ref. 16 and 17, the infrared spectrum of PVAF (Fig. 1a) shows a band at $3270 \mathrm{~cm}^{-1}$ due to the elongation of the $\mathrm{O}-\mathrm{H}$ bonds. The band at $2920 \mathrm{~cm}^{-1}$ could be attributed to the $\mathrm{C}-\mathrm{H}\left(\mathrm{CH}_{3}, \mathrm{CH}_{2}\right)$ bond elongation, this band was characteristic of materials with saturated carbons or $\mathrm{sp}^{3}$. In spectrum of GPTMS/PVAF (Fig. 1b), the band at $912 \mathrm{~cm}^{-1}$ is assigned to the epoxide rings. In addition, the band at around $1020 \mathrm{~cm}^{-1}$ is the characteristic absorption bands of $\mathrm{Si}-\mathrm{O}$ bond. In the spectrum of PVAF-g-PEI (Fig. 1c), the characteristic absorption band of the epoxy groups at $910 \mathrm{~cm}^{-1}$ weaken a lot, whereas the characteristic absorption bands of $\mathrm{N}-\mathrm{H}$ and $\mathrm{C}-\mathrm{N}$ appear at $1565 \mathrm{~cm}^{-1}$ and $1203 \mathrm{~cm}^{-1}$ respectively. The appearances of the bands reveal that PEI has been grafted onto the fiber surface. In the spectrum of PA-PVAF (Fig. 1d), the characteristic absorption band of $\mathrm{C}=\mathrm{N}$ bond appears at $1657 \mathrm{~cm}^{-1}$, indicating that the aldehyde groups of GA have been reacted with the amino groups of PEI. The characteristic absorption peaks appear above fully demonstrates that PA-PVAF has been prepared.

The studied fibers were fixed on the sample table with conductive adhesive, and then coated with platinum. As can be seen in Fig. 2, the surface of the PVA fiber (Fig. 2a) is smooth. The fiber diameter is about $20-40 \mu \mathrm{m}$. Through the grafting reaction of PEI on PVA fibers and subsequent crosslinking modification, the structure of the obtained fibers PA-PVAF (Fig. 2b and c) remains nearly unchanged. However, the surface of the fibers has become a little rough and scraggy after reaction. This may be due to the macromolecular reaction which leading the accumulation of polyethyleneimine molecular chain on the fiber surface, indicating that the polyamine chelating fiber PA-PVAF has been prepared.

Table 1 Elemental analysis of different types of fibers

\begin{tabular}{lccc}
\hline Samples & C (\%) & H (\%) & N (\%) \\
\hline PVAF & 54.73 & 8.68 & - \\
PVAF- -PEI & 55.02 & 9.13 & 2.12 \\
PA-PVAF & 54.83 & 9.35 & 3.46
\end{tabular}
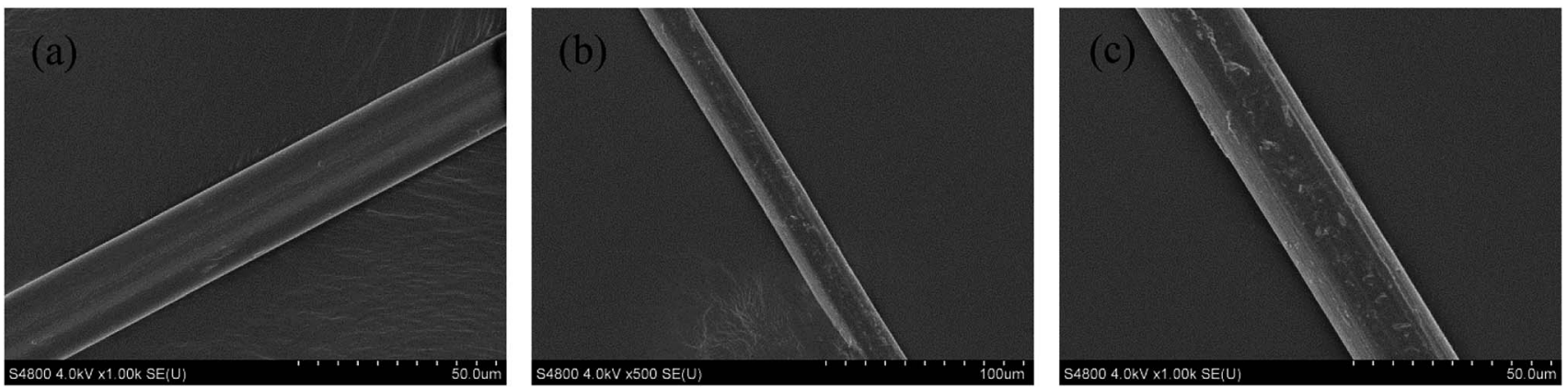

Fig. 2 SEM images of fibers (a) PVAF, (b, c) PA-PVAF. 


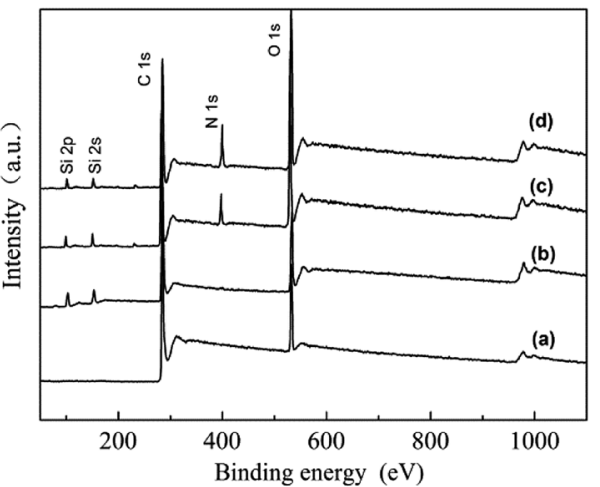

Fig. 3 XPS survey spectra of different fibers (a) PVAF, (b) GPTMS/PVAF, (c) PVAF-g-PEI, (d) PA-PVAF.

The exact amount of various elements in different types of fibers was analyzed by CHN organic element analyzer. The data obtained are presented in Table 1 . The result shows the presence of $\mathrm{C}$ and $\mathrm{H}$ in the PVAF, and the calculations indicate that the amounts of $\mathrm{C}$ and $\mathrm{H}$ are approximately $54.73 \%$ and $8.68 \%$, respectively. For polyethyleneimine-grafted fibers PVAF- $g$-PEI, a nitrogen percentage of $2.12 \%$ is determined, corresponding to the grafted chains PEI. Meanwhile, PA-PVAF presents a nitrogen content of $3.28 \%$. The elevated percentage suggests that TEPA (nitrogen content at about $37 \%$ ) is attached to PVAF$g$-PEI surface.

The chemical environment of the fiber surface was analyzed by using XPS, wherein the light source is monochromatized $\mathrm{Al}$ $\mathrm{K} \alpha(1486.6 \mathrm{eV})$, and the working cavity pressure is better than $6.65 \times 10^{7} \mathrm{~Pa}$. All binding energies were calibrated by $\mathrm{C} 1 \mathrm{~s}$ hydrocarbon peak at $284.8 \mathrm{eV}$. The linear background was used in the analysis process.

Fig. 3 shows the full spectrum of various fibers. ${ }^{18}$ For PVAF (Fig. 3a), the characteristic peaks of C1s and O1s are detected at $284 \mathrm{eV}$ and $399 \mathrm{eV}$ respectively. After modification with GPTMS, the peak of Si2p is detected at $102 \mathrm{eV}$ (Fig. 3b). Then, the peak of $\mathrm{N} 1 \mathrm{~s}$ is detected at $530 \mathrm{eV}$ by grafting PEI on the surface (Fig. 3c). After the surface modification of PVAF-g-PEI with TEPA, the species of element has not changed while the peak intensity of the modified surface changes (Fig. 3d).
Table 2 C (1s) division peak percentage content of fiber surface

\begin{tabular}{llll}
\hline Samples & $\begin{array}{ll}\mathrm{C} 1(\%) \\
\mathrm{C}-\mathrm{C} / \mathrm{C}-\mathrm{H}\end{array}$ & $\begin{array}{l}\mathrm{C} 2(\%) \\
\mathrm{C}-\mathrm{O} / \mathrm{C}-\mathrm{N}\end{array}$ & $\begin{array}{l}\mathrm{C} 3(\%) \\
\mathrm{C}=\mathrm{O} / \mathrm{C}=\mathrm{N}\end{array}$ \\
\hline PVAF & 54.03 & 41.42 & 4.55 \\
PVAF- $g$-PEI & 41.16 & 57.3 & 1.54 \\
PA-PVAF & 42.21 & 50.71 & 7.08
\end{tabular}

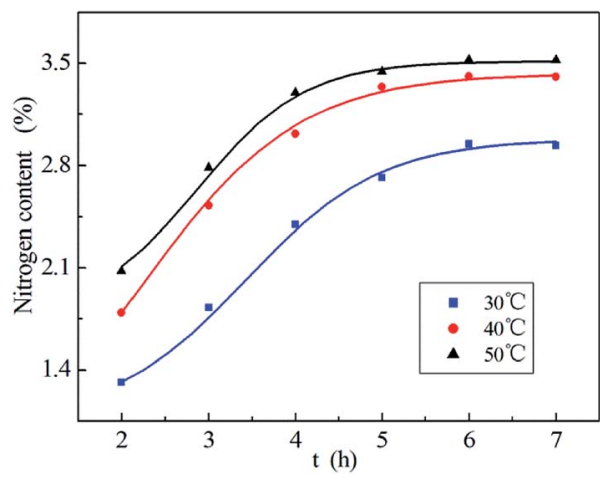

Fig. 5 Variation curves for nitrogen content with time at different temperature.

As shown in Fig. 4 and Table 2, the XPS spectra of C1s is curve-fitted into three individual peaks to represent three types of carbon atoms, including $\mathrm{C} 1(\mathrm{C}-\mathrm{C}, \mathrm{C}-\mathrm{H}), \mathrm{C} 2(\mathrm{C}-\mathrm{O}, \mathrm{C}-\mathrm{N})$ and $\mathrm{C} 3(\mathrm{C}=\mathrm{O}, \mathrm{C}=\mathrm{N}) \cdot{ }^{19,20}$ In the $\mathrm{C} 1 \mathrm{~s}$ spectrum of original fibers PVAF, C1 component is mainly from the carbon skeleton of PVA molecular chain. The $\mathrm{C} 2$ component is due to a carbon bound to hydroxyl oxygen atom. And C3 component is assigned to residual acetyl groups on the molecular chain of PVA. In the C1s spectrum of PVAF- $g$-PEI, as the PEI which contains a large number of amino is adhered on the surface, the percentage content of C2 species increases while that of C3 species decreases. In the C1s spectrum of PA-PVAF, the TEPA is bonded onto the PVAF- $g$-PEI surface by crosslinking reaction with GA. The resultant imine produced by the reaction leads to the increase of C3 species.
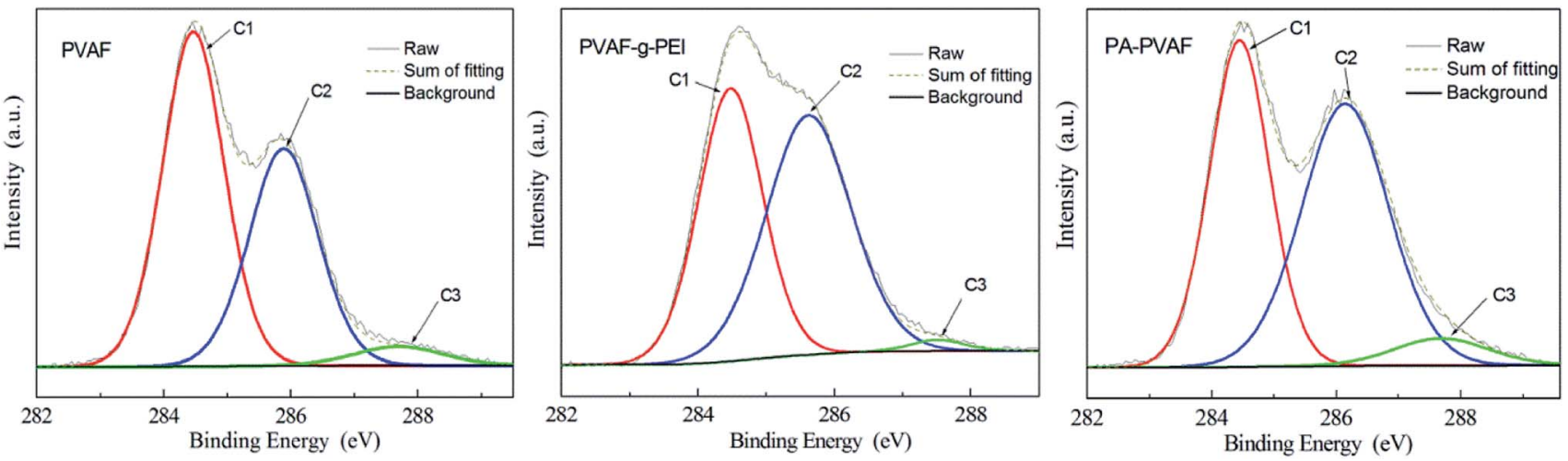

Fig. 4 XPS scan of C1s region for PVAF, PVAF-g-PEI and PA-PVAF. 
Table 3 Effect of reaction conditions on nitrogen content

\begin{tabular}{|c|c|c|c|c|c|}
\hline Entry & $V_{\text {TEPA }}(\mathrm{mL})$ & $V_{\mathrm{GA}}^{a}(\mathrm{~mL})$ & $T\left({ }^{\circ} \mathrm{C}\right)$ & Reaction time (h) & Nitrogen content $(\%)$ \\
\hline 1 & 2.0 & 2 & 40 & 6 & 1.82 \\
\hline 2 & 2.0 & 5 & 40 & 6 & 2.59 \\
\hline 4 & 2.0 & 10 & 40 & 6 & 3.32 \\
\hline 5 & 2.5 & 8 & 40 & 6 & 1.45 \\
\hline 6 & 3.0 & 8 & 40 & 6 & 3.46 \\
\hline
\end{tabular}

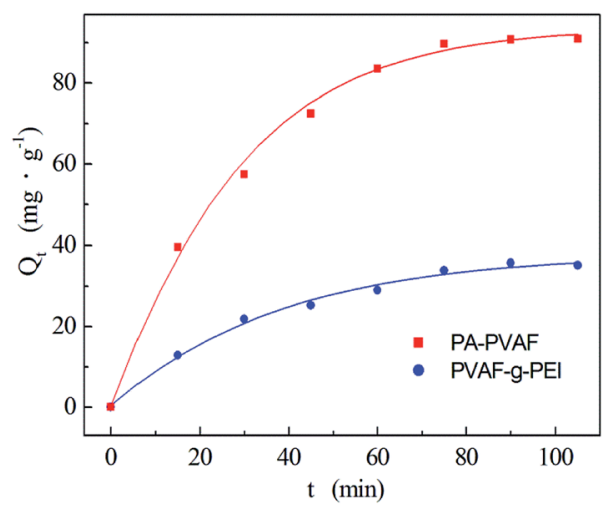

Fig. 6 Effect of contact time on Ni(॥) adsorption by PVAF-g-PEI and PA-PVAF. $C_{0}(\mathrm{Ni}(\mathrm{I})) 300 \mathrm{mg} \mathrm{L}^{-1}$; $\mathrm{pH} 7.0$; temperature $293 \mathrm{~K}$.

\subsection{Influence of the main factors on the preparation of PA- PVAF}

In this study, the effect of reactant ratio, reaction temperature, reaction time and dosage of GA on PA-PVAF preparation were investigated. The preferred conditions for the reaction were selected by measuring the nitrogen content of the products.

By fixing the other reaction condition, the nitrogen content of PA-PVAF varies with time at different temperature are shown in Fig. 5. With the increase of temperature, the nitrogen content increases at the same time. The results indicate that increase of temperature is beneficial to the reaction. Therefore, the suitable reaction temperature and time is $40{ }^{\circ} \mathrm{C}$ and $6 \mathrm{~h}$.

The amount of reactant PVAF-g-PEI was fixed at $1.0 \mathrm{~g}$. As shown in Table 3, the nitrogen content of product PA-PVAF increases by increasing the GA amount. When the dosage of GA is $8 \mathrm{~mL}$, the nitrogen content is up to $3.25 \%$. With further increasing the GA amount, the subsequent addition of GA does not participate in the cross-linking reaction, resulting in little change in nitrogen content.

In addition, it can be observed that the nitrogen content increases with increasing the dosage of TEPA solution. When the addition amount is $3.0 \mathrm{~mL}$, the nitrogen content is up to $3.46 \%$. But the nitrogen content decreases when the amount of TEPA increases continue. The main reason is that when the amount of TEPA solution is too large, the intermolecular crosslinking reaction of TEPA or PEI increased. Therefore, the bonded amount of TEPA decreases and the nitrogen content of product PA-PVAF decreases.

\subsection{Study on adsorption properties of chelating fiber PA-PVAF}

2.3.1 Effect of adsorption time and adsorption kinetics. Fig. 6 illustrates the effect of contact time on the adsorption of Ni(II) using PVAF-g-PEI and PA-PVAF. It can be noted that the adsorption quantity of $\mathrm{Ni}(\mathrm{II})$ increases rapidly and finally attains equilibrium at around $90 \mathrm{~min}$. The higher adsorption rate may be caused by the following factors: the fiber diameter is small $(20-40 \mu \mathrm{m})$ and the effective surface area is large; the chelating adsorption property of the composite fiber is enhanced through the grafting modification of PVA fiber with PEI and TEPA.

Additionally, the maximum amount of Ni(II) adsorption in Fig. 6 is found to be $35.67 \mathrm{mg} \mathrm{g}^{-1}$ for PVAF-g-PEI, and $91.03 \mathrm{mg}$ $\mathrm{g}^{-1}$ for PA-PVAF, respectively. The polyethyleneimine chains on the surface of PVAF- $g$-PEI can adsorb heavy metal ions through the abundant amino. Through the amino-modification of PVAF$g$-PEI with the aid of crosslinking agent GA, the number of nitrogen atoms with lone pairs of electrons on PA-PVAF chains increases, which gives rise to more adsorption sites and leads to greater capacity of adsorption for $\mathrm{Ni}(\mathrm{II})$.

The adsorption kinetic data were fitted with the pseudo first order (eqn (2.1)) and pseudo second order kinetics model (eqn (2.2)) to further study the adsorption kinetics. ${ }^{21,22}$ They

Table 4 Adsorption kinetic constants

\begin{tabular}{|c|c|c|c|c|c|c|c|}
\hline \multirow[b]{2}{*}{ Adsorbent } & \multirow[b]{2}{*}{$Q_{\text {experimental }} /\left(\mathrm{mg} \mathrm{g}^{-1}\right)$} & \multicolumn{3}{|c|}{ Pseudo-first-order model } & \multicolumn{3}{|c|}{ Pseudo-second order model } \\
\hline & & $Q_{\mathrm{e}} /\left(\mathrm{mg} \mathrm{g}^{-1}\right)$ & $k_{1} / \min ^{-1}$ & $R^{2}$ & $Q_{\mathrm{e}} /\left(\mathrm{mg} \mathrm{g}^{-1}\right)$ & $k_{2} /\left(\mathrm{g} \mathrm{mg}^{-1} \min ^{-1}\right)$ & $R^{2}$ \\
\hline PVAF- $g$-PEI & 35.67 & 57.74 & 0.040 & 0.958 & 45.63 & $1.43 \times 10^{-4}$ & 0.988 \\
\hline PA-PVAF & 91.03 & 198.13 & 0.066 & 0.971 & 115.20 & $3.12 \times 10^{-4}$ & 0.991 \\
\hline
\end{tabular}




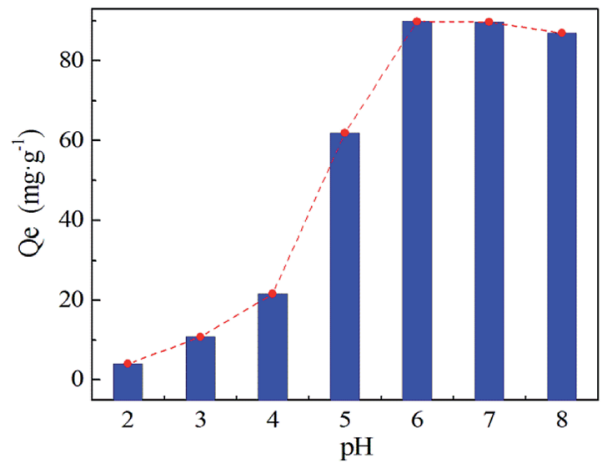

Fig. 7 Effect of initial pH $C_{0}\left(\mathrm{Ni}\left({ }_{1}\right)\right) 300 \mathrm{mg} \mathrm{L}^{-1}$; temperature $293 \mathrm{~K}$.

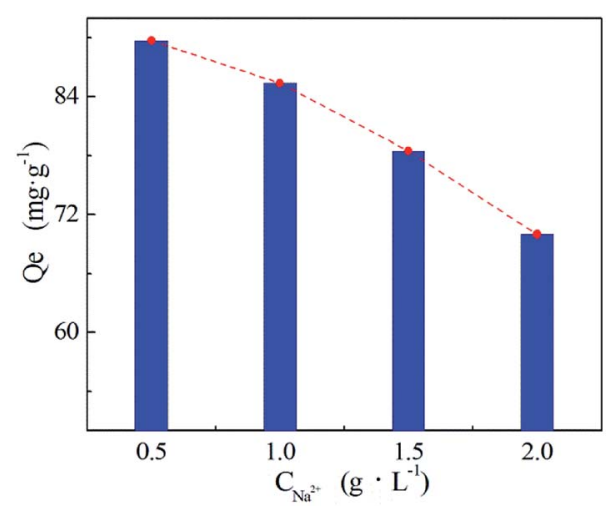

Fig. 8 Effect of ionic strength $C_{0}\left(\mathrm{Ni}\left({ }_{1}\right)\right) 300 \mathrm{mg} \mathrm{L}^{-1}$; $\mathrm{pH}$ 7.0; temperature $293 \mathrm{~K}$.

corresponding kinetic controlling mechanism is assumed of liquid film diffusion and surface chemical adsorption, respectively. The corresponding expressions are as follows:

$$
\begin{gathered}
\ln \left(Q_{\mathrm{e}}-Q_{t}\right)=\ln Q_{\mathrm{e}}-k_{1} t \\
\frac{t}{Q_{t}}=\frac{1}{k_{2} Q_{\mathrm{e}}{ }^{2}}+\frac{t}{Q_{\mathrm{e}}}
\end{gathered}
$$

where $k_{1}, k_{2}$ : equilibrium adsorption rate constant; $Q_{t}, Q_{\mathrm{e}}$ : the adsorption amount of PA-PVAF to $\mathrm{Ni}(\mathrm{II})$ at $t$ moment and adsorption equilibrium.

As shown in Table 4, the correlation coefficient $R^{2}$ of pseudosecond order model is closer to 1 than that of pseudo-first order model. The equilibrium adsorption amount of $Q_{\mathrm{e}}$ obtained by the pseudo-second order model is closer to the experimental value $\left(Q_{\text {test }}\right)$. The adsorption rate $k_{2}$ of PA-PVAF is faster than that of PVAF-g-PEI due to the strong surface chelation. It is shown that the adsorption process can be described by pseudosecond order model, and the adsorption rate is controlled by surface chemical reaction.

2.3.2 Effects of ionic strength and $\mathrm{pH}$ on adsorption properties. The adsorption capacity curve of PA-PVAF for Ni(II) with different $\mathrm{pH}$ and different ionic strength are shown in Fig. 7 and 8, respectively.

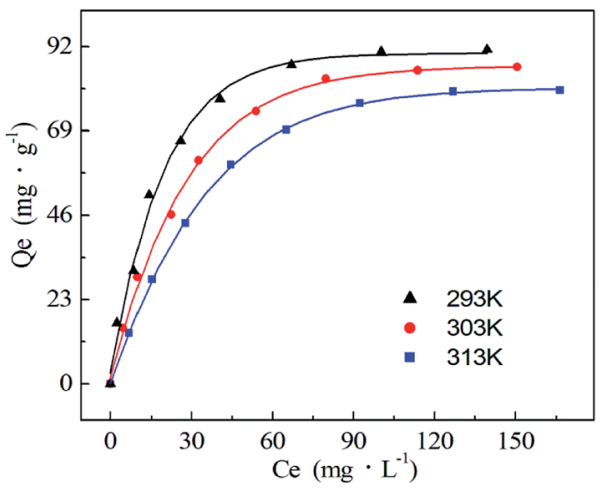

Fig. 9 Adsorption isotherms of $\mathrm{Ni}(I)$ adsorption by PA-PVAF at different temperatures. $C_{0}\left(\mathrm{Ni}\left({ }_{1}\right)\right) 300 \mathrm{mg} \mathrm{L}^{-1}$; $\mathrm{pH} 7.0$.

The solution $\mathrm{pH}$ not only affects the form of heavy metal ions, but also determines the surface charge of the adsorbent. The adsorption behavior of Ni(II) on PA-PVAF surface has been investigated within $\mathrm{pH}$ range of $2.0-8.0$ at an initial $\mathrm{Ni}(\mathrm{II})$ concentration of $300 \mathrm{mg} \mathrm{L}^{-1}$. High value of $\mathrm{pH}$ was not tested due to the precipitation of $\mathrm{Ni}(\mathrm{II})$ as hydroxides..$^{23,24}$ As can be seen in Fig. 7, the adsorption capacity of $\mathrm{Ni}(\mathrm{II})$ is obviously low under low $\mathrm{pH}$. This is because amino groups on the PA-PVAF surface are in the protonation state at low $\mathrm{pH}$. This leads to electrostatic repulsion with the positively charged $\mathrm{Ni}(\mathrm{II})$, reducing the adsorption capacity. With increasing $\mathrm{pH}$, the fiber surface protonation decreases and promotes the adsorption of $\mathrm{Ni}(\mathrm{II})$. When $\mathrm{pH}$ is 7.0 , the equilibrium adsorption capacity of PVAF- $g$-CACTS to Ni(II) reaches $89.70 \mathrm{mg} \mathrm{g}^{-1}$. Moreover, the $\mathrm{pH}$ selected for further experiments is 7.0.

The effect of ionic strength on $\mathrm{Ni}(\mathrm{II})$ adsorption onto PAPVAF was investigated by adding $\mathrm{NaCl}$ at different concentrations (from $0.5 \mathrm{~g} \mathrm{~L}^{-1}$ to $2.0 \mathrm{~g} \mathrm{~L}^{-1}$ ). The initial $\mathrm{Ni}(\mathrm{II})$ concentration in the aqueous solution was $300 \mathrm{mg} \mathrm{L}^{-1}$, the results are illustrated in Fig. 8. It can be seen that the decrease in saturated adsorption quantity with increase in salt concentration. At the highest salt concentration $\left(2.0 \mathrm{~g} \mathrm{~L}^{-1}\right)$, the amounts of adsorbed $\mathrm{Ni}$ (II) decrease to $71.23 \mathrm{mg} \mathrm{g}^{-1}$. That may be due to the high salt concentrations exist in aqueous solution, which not only affects the ionic strength of the water, but also may compete for adsorption sites against heavy metal ions. ${ }^{25}$ The effective adsorption sites of adsorbent PA-PVAF were occupied by $\mathrm{Na}^{+}$, resulting in the reduction of $\mathrm{Ni}(\mathrm{II})$ adsorption.

2.3.3 Effect of ion concentration and adsorption isotherm. As can be seen in Fig. 9, when the initial concentration of Ni(II) increases from 30 to $300 \mathrm{mg} \mathrm{L}^{-1}$, the adsorption capacity of $\mathrm{Ni}(\mathrm{II})$ increases. When the concentration of $\mathrm{Ni}(\mathrm{II})$ ions reaches a certain value, the equilibrium adsorption capacity is almost constant. This is because the adsorption site of surfactant adsorption tends to saturation with increasing the initial concentration. Furthermore, it can be observed from Fig. 9 that the adsorption amount of PA-PVAF towards Ni(II) decreases with the increase of temperature, indicating that the adsorption is an exothermic process. 
Table 5 Langmuir and Freundlich isotherm constants for Ni(I) adsorption

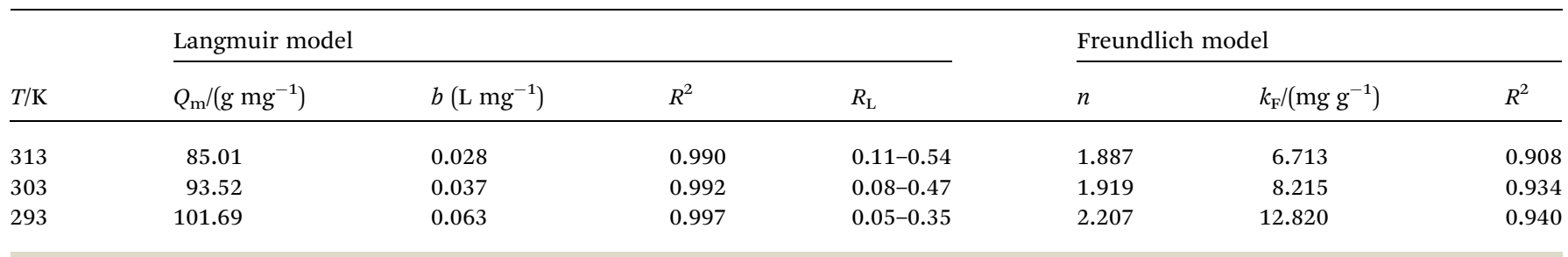

Table 6 Comparison of $\mathrm{Ni}($ II) adsorption with other reported adsorbents

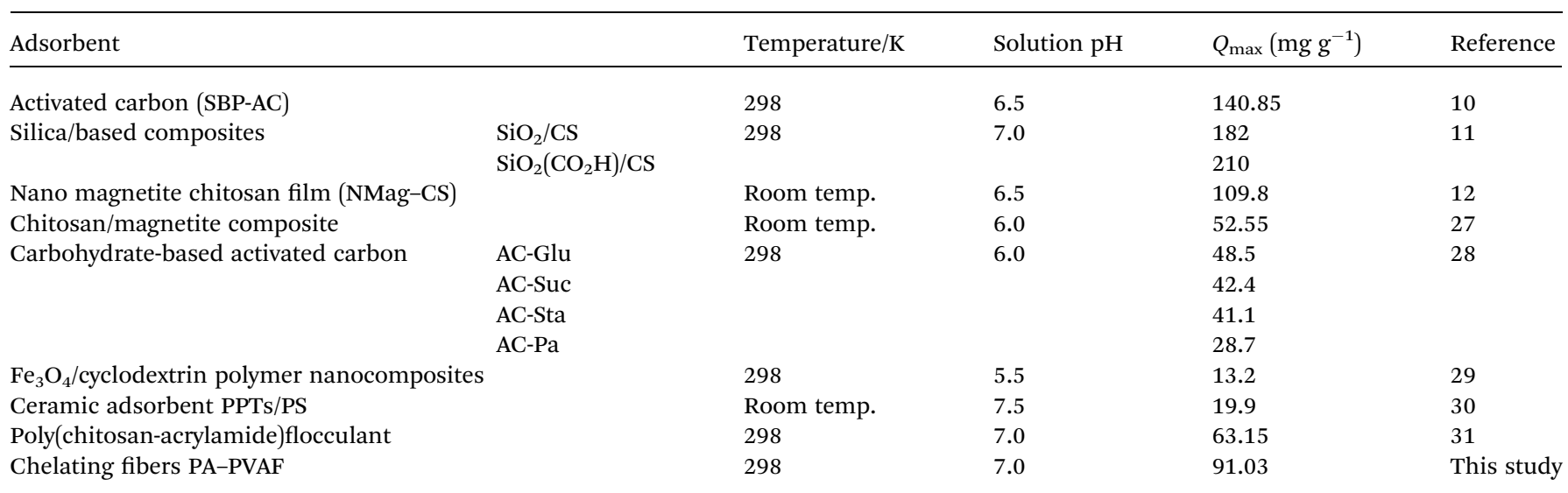

The Langmuir (eqn (2.3)) and Freundlich (eqn (2.4)) isothermal models are used to describe experimental data of adsorption isotherm..$^{25,26}$ The Langmuir isotherm assumes monolayer coverage of the adsorption surface, and no further adsorption can occur when the adsorption saturates; the Freundlich isotherm describes the adsorption of the polymer layer at the heterogeneous interface. The corresponding expressions are as follows:

$$
\frac{C_{\mathrm{e}}}{Q_{\mathrm{e}}}=\frac{1}{b Q_{\mathrm{m}}}+\frac{C_{\mathrm{e}}}{Q_{\mathrm{m}}}
$$

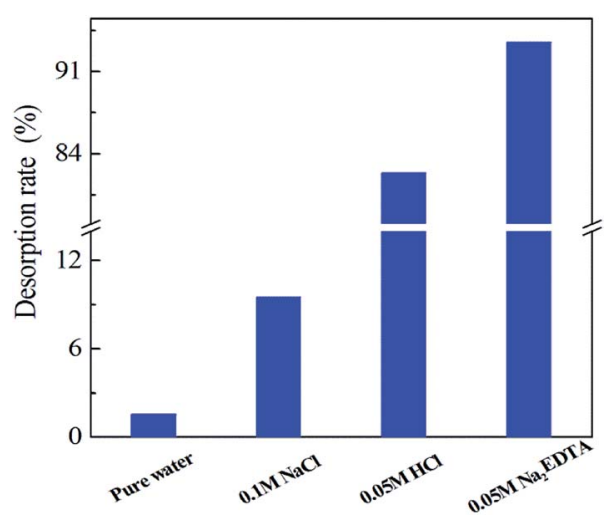

Fig. 10 Desorption rate of $\mathrm{Ni}($ II) from PA-PVAF using different desorption eluents $C_{0}(\mathrm{Ni}(\mathrm{II})) 300 \mathrm{mg} \mathrm{L}^{-1}$; $\mathrm{pH}$ 7.0; temperature $293 \mathrm{~K}$.

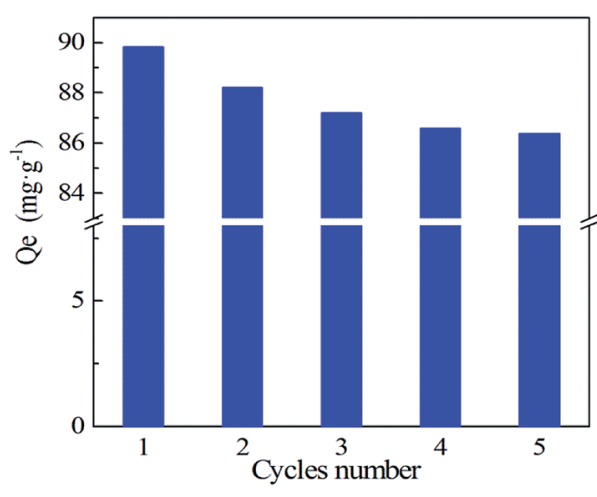

Fig. 11 Five adsorption-desorption cycles of PA-PVAF adsorbent for $\mathrm{Ni}\left(\right.$ II) $\mathrm{C}_{0}\left(\mathrm{Ni}\left(\right.\right.$ II)) $300 \mathrm{mg} \mathrm{L}^{-1}$; pH 7.0; temperature $293 \mathrm{~K}$.

$$
\ln Q_{\mathrm{e}}=\ln k_{\mathrm{F}}+\frac{\ln C_{\mathrm{e}}}{n}
$$

where $C_{\mathrm{e}}$ is the equilibrium concentration; $Q_{\mathrm{e}}$ the equilibrium adsorption capacity; $Q_{\mathrm{m}}$ the maximum adsorption capacity; $k_{\mathrm{F}}$ and $1 / n$ are Freundlich constants.

Table 5 lists the parameters derived from the Langmuir and Freundlich models. It can be seen that the values of $R^{2}$ for Langmuir model are exceedingly high in comparison to value of $R^{2}$ for Freundlich model. It is obvious that the data were fitted to the Langmuir model. Isotherm with $n>1$ is implying a high affinity between PA-PVAF and $\mathrm{Ni}(\mathrm{II})$ and is indicative of chemisorption. $^{12}$ 
At the same time, the applicability of the Langmuir model can be articulated according to the dimensionless parameter, $R_{\mathrm{L}}$ (eqn (2.5)). Where $b$ is the Langmuir constant and $C_{0}$ is the initial concentration of $\mathrm{Ni}(\mathrm{II})$. The value of $R_{\mathrm{L}}$ reveals whether the type of isotherm adsorption is unfavorable $\left(R_{\mathrm{L}}>1\right)$, favorable $\left(0<R_{\mathrm{L}}<1\right)$, irreversible $\left(R_{\mathrm{L}}=0\right)$ or linear $\left(R_{\mathrm{L}}=1\right) \cdot{ }^{26}$ As can be seen from Table 5 , the values of $R_{\mathrm{L}}$ over an initial Ni(II) concentration range of $30-300 \mathrm{mg} \mathrm{L}^{-1}$ at different temperature were between 0 and 1 , which indicated that the experimental conditions were favorable for adsorption.

$$
R_{\mathrm{L}}=\frac{1}{\left(b C_{0}+1\right)}
$$

Compared with other adsorbents reported in literature, the polyamine chelating fiber PA-PVAF exhibits better adsorption performance than most reported ones (Table 6). The difference in maximum adsorption capacity $\left(Q_{\max }\right)$ is most likely caused by various functional groups, different types of structures, and diverse adsorption conditions.

\subsection{Regeneration and reusability}

The desorption of Ni(II) from the PA-PVAF was carried out with distilled water, $0.1 \mathrm{M} \mathrm{NaCl}, 0.05 \mathrm{M} \mathrm{NaCl}$ and $0.05 \mathrm{M} \mathrm{Na}_{2}$ EDTA, and the corresponding desorption rate was $1.65 \%, 9.52 \%$, $82.36 \%$ and $93.39 \%$, respectively (Fig. 10). The results revealed that $0.05 \mathrm{M} \mathrm{Na}_{2}$ EDTA was a more suitable eluent. The adsorption-desorption experiments were carried out for 5 times, and the reusability versus recycling time is shown in Fig. 11. It can be seen that the adsorption capacity of PVAF-g-CACTS to Cd(II) decreases only slightly after 5 cycles. The adsorption capacity in the fifth cycle is still $86.35 \mathrm{mg} \mathrm{L}^{-1}$. It proves that PA-PVAF shows excellent performance of reuse.

\section{Conclusions}

A novel polyamine chelating fiber material PA-PVAF was prepared with crosslinking PEI and TEPA using GA as crosslinker. The optimal reaction conditions for the preparation of PA-PVAF were investigated. The appropriate reaction temperature is $40^{\circ} \mathrm{C}$ and the suitable reaction time is $6 \mathrm{~h}$. Moreover, the adsorption property of PA-PVAF to $\mathrm{Ni}$ (II) was evaluated. The maximum adsorption of $\mathrm{Ni}(\mathrm{II})$ was observed at $\mathrm{pH}$ 7.0, and the adsorption amount can reach up to $91.03 \mathrm{mg} \mathrm{g}^{-1}$. The adsorption capacity decreased with the increase of temperature, indicating that the adsorption of $\mathrm{Ni}(\mathrm{II})$ ions on PA-PVAF was spontaneous. The Langmuir isotherm model precisely explained the equilibrium data. Adsorbent-adsorbate kinetics exhibited pseudo second order. $0.05 \mathrm{M} \mathrm{Na}_{2}$ EDTA solution showed excellent desorption efficiency for $\mathrm{Ni}(\mathrm{II})$ with a recovery of $92.6 \%$. The adsorption-desorption cycle experiments showed that PA-PVAF had good performance of reuse. After five cycles, the adsorption capacity of PA-PVAF towards Ni(II) still reached $86.35 \mathrm{mg} \mathrm{L}^{-1}$. Apart from its great performance for the retention of $\mathrm{Ni}(\mathrm{II})$, the new sorbents presented additional advantages such as simple technique and facile preparation.

\section{Acknowledgements}

We are grateful to National Natural Science Foundation of China (Project No. 22404093), Fund for Postgraduate of North University of China (Project No. 20151230).

\section{Notes and references}

1 P. Z. Ray and H. J Shipley, RSC Adv., 2015, 5, 29885-29907.

2 O. Ozay, S. Ekici, Y. Baran, N. Aktas and N. Sahiner, Water Res., 2009, 43, 4403-4411.

3 H. H. Najafabadi, M. Irani, L. R Rad, A. H Haratameh and I. Haririan, RSC Adv., 2015, 5, 16532-16539.

4 S. T. Yang, G. D Sheng, X. L. Tan, J. Hu, J. Z. Du, G. Montavon and X. K. Wang, Geochim. Cosmochim. Acta, 2011, 75, 65206534.

5 C. E. Borba, R. Guirardello, E. A. Silva, M. T. Veit and C. R. G. Tavares, Biochem. Eng. J., 2006, 30, 184-191.

6 A. H. Gedam and R. S. Dongre, RSC Adv., 2015, 5, 5418854201.

7 C. Santhosh, V. Velmurugan, G. Jacob, S. K. Jeong, A. N. Grace and A. Bhatnagar, Chem. Eng. J., 2016, 306, 1116-1137.

8 M. Xu, Y. S Zhang, Z. M Zhang, Y. Shen, M. J Zhao and G. T Pan, Chem. Eng. J., 2011, 168, 737-745.

9 T. Budinova, D. Savova and B. Tsyntsarski, Appl. Surf. Sci., 2009, 255, 4650-4657.

10 K. Anoop Krishnan, K. G. Sreejalekshmi and R. S. Baiju, Bioresour. Technol., 2011, 102, 10239-10247.

11 R. Singhon, J. Husson, M. Knorr, B. Lakard and M. Euvrard, Colloids Surf., B, 2012, 93, 1-7.

12 M. R. Lasheen, I. Y. El-Sherif, M. E. Tawfik, S. T. El-Wakeel and M. F. El-Shahat, Mater. Res. Bull., 2016, 80, 344-350.

13 A. Denizli, S. Senel, G. Alsancak, N. Tuzmen and R. Say, React. Funct. Polym., 2003, 55, 121-130.

14 B. J. Gao, F. Q. An and K. K. Liu, Appl. Surf. Sci., 2006, 253, 1946-1952.

15 A. K. Thakura, G. M. Nisolaa, L. A. Limjucoa, K. J. Parohinog, R. E. C. Torrejos, V. K. Shahi and W. J. Chung, J. Ind. Eng. Chem., 2017, 49, 133-144.

16 Y. Shirosaki, K. Tsuru, S. Hayakawa, A. Osaka, M. A. Lopes, J. D. Santos, M. A. Costa and M. H. Fernandes, Acta Biomater., 2009, 5, 346-355.

17 S. Pouranvari, F. Ebrahimi, G. Javadi and B. Maddah, Mater. Tehnol., 2016, 50(5), 663-666.

18 S. Altenor, B. Carene, E. Emmanuel, J. Lambert, J. J. Ehrhardt and S. Gaspard, J. Hazard. Mater., 2009, 165, 1029-1039.

19 G. X. Zhang, S. H. Sun, D. Q. Yang, J. P. Dodelet and E. Sacher, Carbon, 2008, 46, 196-205.

$20 \mathrm{H}$. Chen and Z. Hashisho, Fuel, 2012, 95, 178-182.

21 Y. Cheng, C. P. Yang, H. J. He, G. M. Zeng, K. Zhao and Z. Yan, J. Environ. Eng., 2016, 142, C4015001.

22 C. Yang, J. Q. Wang, M. Lei, G. X. Xie, G. M. Zeng and S. L. Luo, J. Environ. Sci., 2010, 22(5), 675-680.

23 Y. Vijaya, S. R. Popuri, V. M. Boddu and A. Krishnaiah, Carbohydr. Polym., 2008, 72, 261-271. 
24 E. Ahmet, N. Tirtom, T. Aydemir, S. Becerik and A. Dincer, Chem. Eng. J., 2012, 210, 590-596.

25 Y. Q. Huang, C. P. Yang, Z. C. Sun, G. G. Zeng and H. J. He, RSC Adv., 2015, 5, 11475-11484.

26 R. Sharma, A. Sarswat, C. U. Pittman Jr and D. Mohan, RSC Adv., 2017, 7, 8606-8624.

27 H. V. Tran, L. D. Tra and T. N. Nguyen, Mater. Sci. Eng., C, 2010, 30, 304-310.

28 H. Liu, J. Zhang, H. H. Ngo, W. S. Guo, H. M. Wu, C. Cheng, Z. Z. Guo and C. L. Zhang, RSC Adv., 2015, 5, 52048-52056.
29 A. Z. M. Badruddozaa, Z. B. Z. Shawona, T. W. D. Tay, K. Hidajat and M. S. Uddin, Carbohydr. Polym., 2013, 91, 322-332.

30 M. A. A Gonzalez, A. A. Z. Cadena, C. N. Aguilar and E. M. Muzquiz, RSC Adv., 2015, 5, 29748-29756.

31 A. S. Saleh, A. G. Ibrahim, F. Abdelhai, E. M. Elsharma, E. Metwally and T. Siyam, Radiat. Phys. Chem., 2017, 134, 33-39. 